\title{
Vortex shedding control of circular cylinder by perforated shroud in deep water
}

\author{
Gokturk M. Ozkan ${ }^{1}$, Tahir Durhasan ${ }^{1 *}$,Engin Pinar $^{2}$, Muhammed M. Aksoy ${ }^{3}$, Huseyin Akilli ${ }^{1}$ and Beşir Sahin ${ }^{1}$ \\ ${ }^{1}$ Cukuruova University, Mechanical Engineering Department, 01330 Adana, Turkey \\ ${ }^{2}$ Cukuruova University, Ceyhan Mechanical Engineering Department, 01950 Adana, Turkey \\ ${ }^{3}$ Osmaniye Korkut Ata University, Energy Systems Department, 80000 Osmaniye, Turkey
}

\begin{abstract}
The aim of the present study is to control the vortex shedding downstream of a circular cylinder (inner cylinder) by the existence of outer perforated cylinder concentrically located around the inner cylinder in deep water. The flow characteristics downstream of concentrically placed coupled cylinders were investigated quantitatively by the Particle Image Velocimetry (PIV) technique. Diameter of the outer perforated cylinder and inner cylinder were kept constant as $\mathrm{D}_{0}=100 \mathrm{~mm}$ and $\mathrm{D}_{\mathrm{i}}=50 \mathrm{~mm}$. The depthaveraged free-stream velocity was also kept constant as $U=100 \mathrm{~mm} / \mathrm{s}$ which corresponded to the Reynolds number of $R_{\text {Do }}=10,000$ based on the outer cylinder diameter. Experiments were conducted for six porosities $(\beta=0.3,0.4,0.5,0.6,0.7$ and 0.8$)$ in order to show the effect of these parameters on the flow control. Maximum values of both Reynolds shear stress, $<\mathrm{uv}>$ and turbulence kinetic energy, $<$ TKE $>$ significantly decreased with the existence of outer perforated cylinder and also, the location of peak magnitudes of turbulence statistics occurred at locations further downstream compared to the bare cylinder case. The most effective control was revealed for the porosity of $\beta=0.7$.
\end{abstract}

\section{Introduction}

It is well-known phenomena that flow over a circular cylinder which causes acoustic noise, vibration, fatigue and shortens the life of cylinder. To end this, the flow control around a cylinder has been an attractive topic for more than a century due to its engineering significance and in part due to its tempting simplicity in setting up arrangements in experimental or numerical studies.

Ikeda and Takaishi [1] showed that the stable wake shear layers of the perforated cylinder and suppression of Aeolian tone are achieved due to the jets emitted from the holes at regular intervals. Boorsma et. al. [2] performed experimental study to investigate and optimize the effect of different perforated fairing on the noise control at different location. The fairing self-noise is reduced remarkably by breakdown of the vortex shedding process, resulting in a reduction of associated broadband noise level. Zho and Cheng [3] demonstrated that lift reduction of circular cylinder can be achieved by properly choosing the porous material. Kleissl and Georgakis [4] performed experimental study in order to show the effect of modifying bridge cable shape and surface on suppression of the vortex induced vibration. They revealed that shroud cylinder significantly reduced the vortex-induced oscillating lift forces. Yu et al. [5] studied numerically on the steady flow around a porous circular cylinder. They found that contrary to that of the solid cylinder, the recirculating wake develops downstream of or within the porous cylinder, but not from the surface of it.Ozkan et al. [6] expressed that the change in flow characteristics downstream of circular cylinder surrounded by a permeable cylinder in shallow water using particle image velocimetry technique. They observed that the peak magnitude of turbulent kinetic energy and Reynolds stress decrease remarkably due to the presence of the outer cylinder compare with natural cylinder case. Gozmen and Akilli [7] studied on the control of flow around cylinder surrounded by permeable cylinder in deep water. They noted that parameters, which are the porosity and diameter ratio, play important role on the suppression of vortex shedding. Pinar et al. [8] experimentally investigated flow structure of perforated cylinder in shallow water. The results of their study show that porosity had a significant effect on the control of large-scale vortical structures downstream of cylinder. The effect of perforated fairing on the vortex street suppression of circular cylinder was studied by Durhasan et al. [9]. Their results indicated that the wake region of cylinder was elongated remarkably along the main flow direction and vortex shedding frequency reduced substantially by the presence of perforated fairing for porosity in the range of $\beta=0.3-0.6$.

An experimental study was carried out for purpose of controlling the vortex shedding of the circular cylinder using perforated shroud in deep water. The velocity field of downstream of the circular cylinder were determined by using PIV measurement technique.

\footnotetext{
* Corresponding author: tdurhasan@cu.edu.tr
} 
Flow structures and turbulent statistics were analyzed and also compared to bare cylinder in detail.

\section{Experimental setup}

Experiments were carried out in a low turbulence closedloop, opened surface water channel test section having the following dimensions: a length of $8000 \mathrm{~mm}$, width of $1000 \mathrm{~mm}$ and depth of $750 \mathrm{~mm}$. This facility was built in the Fluid Mechanics Laboratory at Cukurova University.

In the present study, perforated outer cylinders constructed from a chrome-nickel steel sheet having 1 $\mathrm{mm}$ thickness. The diameter of outer perforated cylinder and inner cylinder were kept constant as $D_{0}=100 \mathrm{~mm}$ and $\mathrm{D}_{\mathrm{i}}=50 \mathrm{~mm}$, respectively. Six different outer cylinder $(\beta=0.3,0.4,0.5,0.6,0.7$ and 0.8$)$ were used in order to investigate effect of porosity of the perforated shroud. The porosity ratio, $\beta$ was defined as the ratio of the total open area on cylinder to whole cylinder surface area. During the experiments perforated shrouds were located concentrically around the inner cylinder in shown figure 1 . Free stream velocity was $U=100 \mathrm{~mm} / \mathrm{s}$, corresponding Reynolds number $\mathrm{Re}=10000$ based on the outer cylinder diameter.
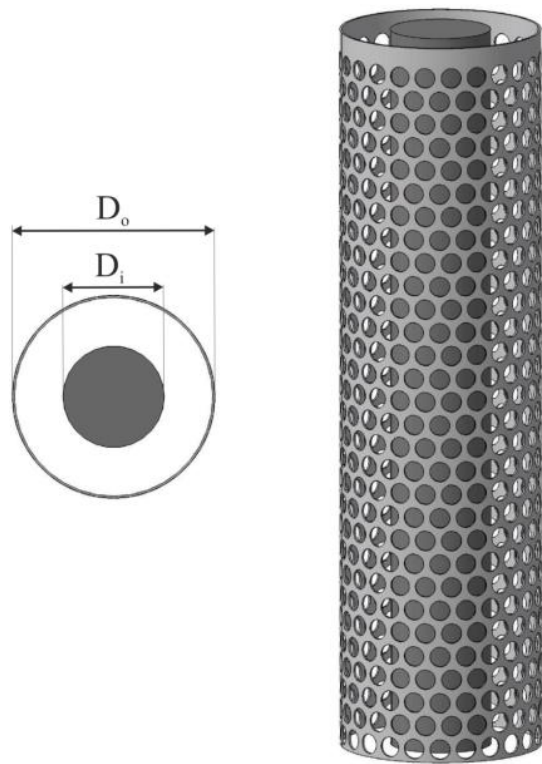

Fig. 1. Schematically representation of perforated shroud

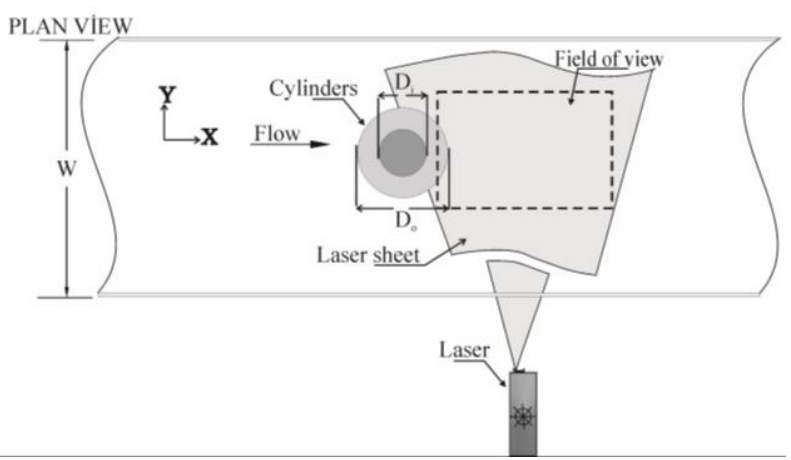

Fig. 2. Schematic view of test section
All measurement were carried out on the plain view for water height of $400 \mathrm{~mm}$ and laser sheet was located parallel to the bottom water channel at height of 200 $\mathrm{mm}$. A schematic view of test section is shown in figure 2.

The experiments were performed and the measured data were processed using Dantec Dynamics PIV system and FLOW MANAGER Software installed on a computer. The illumination of measurement field was supplied with a thin and an intense laser light sheet by using a pair of double-pulsed Nd:YAG (yttrium aluminum garnet) laser source energy level of $120 \mathrm{~mJ}$ at $532 \mathrm{~nm}$ wavelength. The time interval between pulses was $1.75 \mathrm{~ms}$ for all experiments and the thickness of the laser sheet was approximately $2 \mathrm{~mm}$. The time interval and the laser sheet thickness were selected such that the maximum amount of particles in the interrogation window was obtained. The image capturing was performed by an 8 bit cross-correlation charge-coupled device (CCD) camera having a resolution of $1016 \times 1016$ pixels, equipped with a Nikon AF Micro $60 f / 2.8 \mathrm{D}$ lenses. In the image processing, $32 \times 32$ integration window pixels were used and an overlap of $50 \%$ was employed. A total of $3844(62 \times 62)$ velocity vectors were obtained for an instantaneous velocity field at a rate of 15 frames / s. The field of view of measuring planes covers the downstream of the cylinder with the area of $200 \mathrm{~mm} \times 200 \mathrm{~mm}$ for all configurations and the image magnification was $0.0709 \mathrm{~mm} /$ pixel. The water was seeded with $12 \mu \mathrm{m}$ diameter hollow glass sphere particles. The number of particles in an interrogation area was in between 20 and 25. The uncertainty in velocity relative to depth averaged velocity is about $2 \%$ in the present experiments. The instantaneous images were captured, recorded, and stored in order to obtain averaged-velocity vectors and other statistical properties of the flow field. Spurious velocity vectors (less than $3 \%$ ) were removed using the local median-filter technique and replaced by using a bilinear least squares fit technique between surrounding vectors.

\section{Results}

First of all, the wake region of bare (inner) cylinder is present in fig. 3 in order to comparison with the controlled situations. Minimum and incremental values of time averaged vorticity $\langle\omega\rangle$ were taken as \pm 2 and 2 , respectively. The solid and dashed lines present positive (counter-clockwise) and negative (clockwise) contours, respectively. Both positive and negative contours of the time averaged vortices were emanated from the lower and upper shoulders of the cylinder and get close to each other at near wake region for bare cylinders. This results present a well-known situation which was called von Karman vortex street. On the other hand, the minimum and incremental values of turbulent kinetic energy, $<$ TKE $>$ contours were taken as 0.01 . Turbulent kinetic energy, $<$ TKE $>$ contours of bare cylinder clearly indicate that flow around the cylinder causes turbulence in the near wake region of bare (inner) cylinder. 


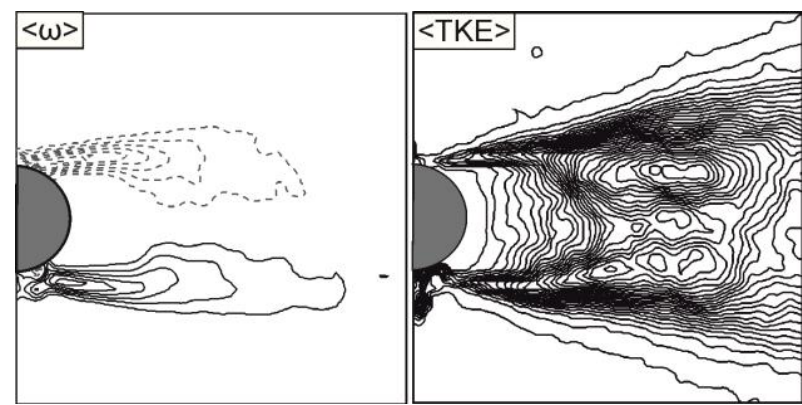

Fig. 3. Distribution of time averaged vorticity, $<\omega>$ and $<\mathrm{TKE}>$ counters for bare cylinder.

Fig. 4. show that distribution of time averaged vorticity counters for different porosity of perforated shroud. Almost symmetrical shear layer structure can be observed for all porosity ratios. It is observed that presence of perforated shroud affects the formation of shear layers on the both sides of cylinder which are gradually elongated with the increasing porosity of the shroud, so that interaction of the shear layer is delayed in the downstream direction. Also, strength of shear layer is decreased with the increasing porosity compared with bare cylinder case. Effect of the perforated shrouds are more dominant on the flow structure downstream of the cylinder in the range of $0.5 \leq \beta \leq 07$. On the other hand, vorticity clusters occur in the near wake region for porosity value of $\beta=0.8$ and this indicated that the perforated have insignificant effect on the flow control for porosity value of $\beta=0.8$.

Time averaged turbulent kinetic energy, $<$ TKE $>$ contours are presented in fig. 5 for different porosity of perforated shroud. Porosity, $\beta$, has a significant effect on the flow structure compared to bare cylinder. Especially, porosity of $\beta \leq 0.6$, TKE intensity along the shear layers decrease with the increasing porosity, $\beta$, and interact with each other in the downstream region at the end of second field of view and peak magnitudes approximately located $7 \mathrm{D}_{\mathrm{i}}$ of the cylinder away from the inner cylinder base. The flow emanating through the holes on the perforated shroud causing a jet flow prevents the vorticity on each side of the coupled cylinder by intercepting the interaction of vortices and thus momentum transfer increases into the wake region. As a result, TKE values in the wake region decrease in comparison to the bare cylinder case. The reason of this kinetic energy intensification in shear layers is the result of prevention of Karman vortices; however, the KelvinHelmholtz vorticity becomes more dominant on the shear layers between the free-stream region and the wake region. Therefore, the most effective flow control is achieved for $\beta=0.7$. Moreover, the peak magnitude of TKE value of $D_{i}=50 \mathrm{~mm}$ bare cylinder is found to be 0.2886 and attenuation rate of the maximum value of TKE is approximately $\% 68$ at most, compared to bare cylinder.

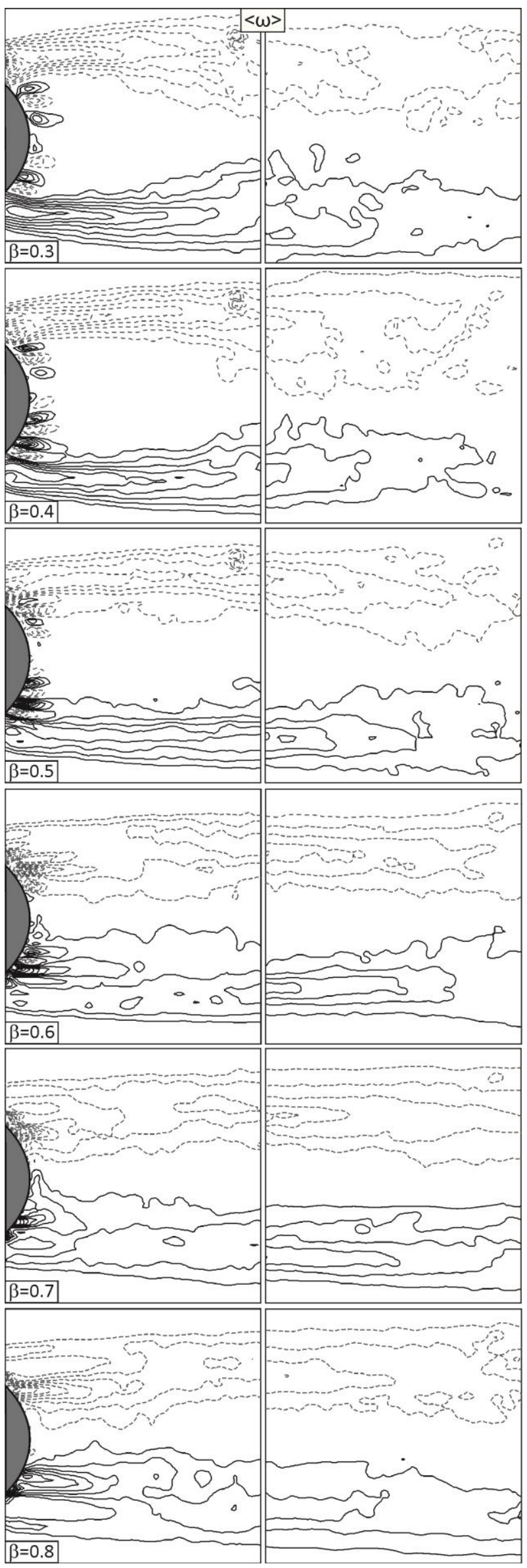

Fig. 4. Distribution of time averaged vorticity, $\langle\omega\rangle$ counters for different porosity of perforated shroud. 


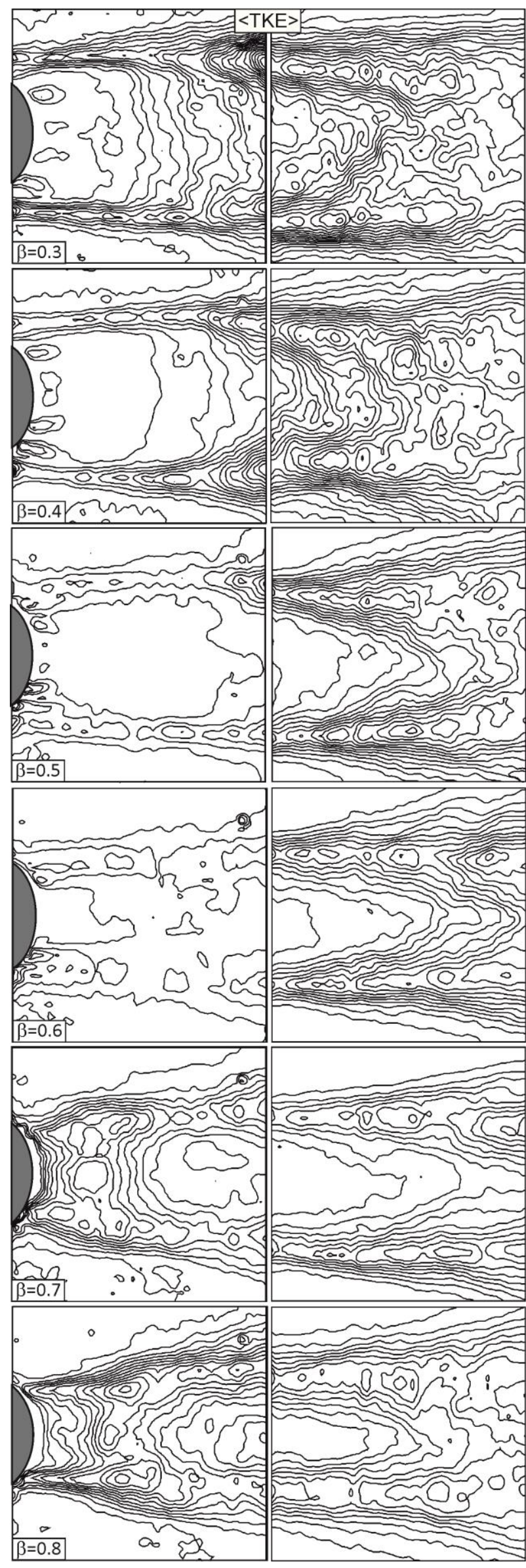

Fig. 5. Distribution of time averaged turbulent kinetic energy, $<$ TKE $>$ counters for different porosity of perforated shroud.
The variation of peak magnitude of Reynolds shear stress, $<u v>$ with respect to porosity, $\beta$ are presented in fig.6. It is observed that the peak magnitude of Reynolds shear stress, $<$ uv $>$ gradually decreased until the porosity value of $\beta=0.7$. Maximum reduction of the peak magnitude of Reynolds shear stress, $<u v>$ is obtained for porosity value of $\beta=0.7$ and it is reduced by $\% 68$ for in compared with bare (inner) cylinder. On the other hand, the peak magnitude of Reynolds shear stress, $<u v>$ for porosity of $\beta=0.8$ is increased to compare with porosity of $\beta=0.7$, but it has still lower value of the peak magnitude of Reynolds shear stress, $<$ uv $>$ than value of bare cylinder case.

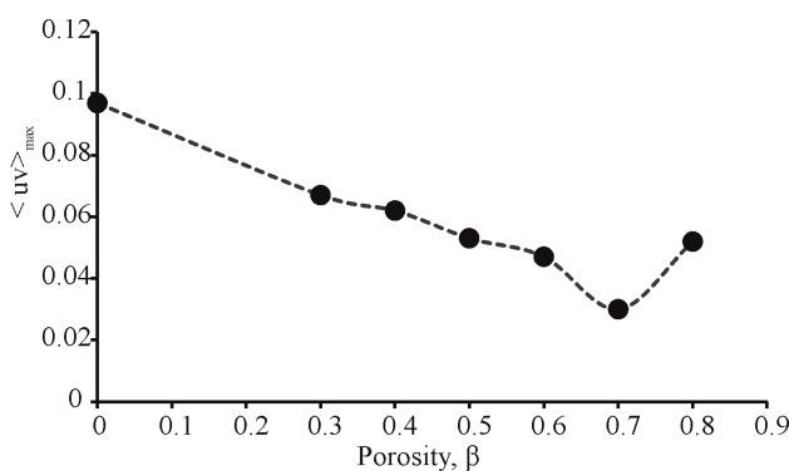

Fig.6. The variation of Reynolds shear stress, $<$ uv $>$ with porosity

\section{Conclusion}

In this study a passive flow control method was applied in order to suppression of vortex shedding downstream of circular cylinder by the existence of perforated shroud concentrically located around the inner cylinder in deep water. To reveal the effect of perforated shroud on the vortex shedding control six different porosity, $\beta$ were used as $\beta=0.3,0.4,0.5,0.6,0.7$ and 0.8 .

It was observed that perforated shroud has a significant effect on the flow structure of circular cylinder. Especially porosity value of $\beta<0.7$, the shear layers of cylinder are elongated gradually due to jet-alike flow through the holes on surface of perforated shroud. Also, turbulence statics are reduced by $\% 68$ for porosity of $\beta=0.7$ in compared with bare cylinder.

\section{Acknowledgements}

This study supported by the funding of the Scientific and Technological Research Council of Turkey under contract no. 109R001.

\section{References}

1. M. Ikeda, T. Takaishi, Perforated pantograph horn aeolian tone suppression mechanism, Q. Report of RTRI (Jpn), 45, 169-174 (2004)

2. K. Boorsma, X. Zhang, N. Molin, L. C. Chow, Bluff body noise control using perforated fairings, AIAA J., 47 33-43 (2009) 
3. M. Zhao, L. Cheng, Finite element analysis of flow control using porous media, Ocean Eng., 37 13571366 (2010)

4. K. Kleissl, C. T. Georgakis, Aerodynamic control of bridge cables through shape modification: A preliminary study, J. Fluids Struc., 27 1006-1020 (2011)

5. P. Yu, Y. Zeng, T. S. Lee, X. B. Chen, H. T. Low, Steady flow around and through a permeable circular cylinder, Comp. Fluids, 42 1-12 (2011)

6. G. M. Ozkan, V. Oruc, H. Akilli, B. Sahin, Flow around a cylinder surrounded by a permeable cylinder in shallow water, Exp. Fluids, 53 17511763 (2012)

7. B. Gozmen, H. Akilli, Flow control downstream of a circular cylinder by a permeable cylinder in deep water, Wind Struc. Int. J., 19 389-404 (2014)

8. E. Pinar, G. M. Ozkan, T. Durhasan, M. M. Aksoy, H. Akilli, B. Sahin, Flow structure around perforated cylinders in shallow water, J. Fluids Struc., 55 52-63 (2015)

9. T. Durhasan, M. M. Aksoy, E. Pinar, G. M. Ozkan, H. Akilli, B. Sahin, Vortex street suppression of a circular cylinder using perforated semi-circular fairing in shallow water, Exp. Term. Fluid Sci, 79 101-110 (2016) 